\title{
On the Convergence Properties of the Quadrilateral Elements of Sander and Beckers*
}

\author{
By Zhong-ci Shi
}

\begin{abstract}
A class of nonconforming quadrilateral membrane elements, introduced by Sander and Beckers, is discussed in all details. It is proved that two elements that do not pass the patch test may still yield convergence under a suitable condition on mesh subdivisions, whereas one such element is found to be divergent for all mesh subdivisions. All other finite elements in this class that pass the patch test are convergent. The mathematical analysis provides a clear explanation of the convergence behavior appearing in the numerical examples of Sander and Beckers.
\end{abstract}

1. Introduction. Quadrilateral finite elements are attractive for discretizations of domains of arbitrary shapes, but these approximations are hardly conforming. Therefore, such elements have to be tested for convergence. In connection with a variational interpretation of the patch test [2], Sander and Beckers [3] have recently introduced a class of nonconforming quadrilateral membrane elements, having 8 to 16 degrees of freedom, and have shown by numerical computations for a trapezoidal membrane problem that two elements that do not pass the patch test still exhibit convergence and yield good approximations, whereas one such element diverges. This result is of great interest and leads to the question whether the patch test is necessary for convergence of these nonconforming elements. As stated in [3], additional investigations are certainly required to understand their behavior.

The present paper establishes a detailed mathematical analysis of these elements. We formulate a condition on mesh subdivisions, under which two elements, that do not pass the patch test, indeed yield convergence, whereas another element is proved to be divergent whatever the mesh subdivision may be. All other quadrilateral elements in this class that pass the patch test are convergent.

The theoretical analysis clearly explains the convergence behavior discussed in the numerical computations of Sander and Beckers. In their examples a bisection scheme of mesh subdivisions is used for the discretization of the trapezoidal membrane. In such cases the proposed condition on the mesh subdivisions is automatically fulfilled, and thus convergence occurs for two elements. It is seen, therefore, that the range of applicability of the patch test as a necessary condition for convergence is limited. At this point it is worth noting that Stummel [5] has presented basic examples which show that spaces of nonconforming trial functions

\footnotetext{
Received May 24, 1983.

1980 Mathematics Subject Classification. Primary 65N30.

* This work was written while the author was visiting the University of Frankfurt, Federal Republic of Germany, on a grant by the Alexander von Humboldt Foundation.
} 
may pass the patch test but still fail to converge. An explicit analysis of these examples is given in [4].

We also discuss the problem of unisolvence of the shape functions of the quadrilateral elements considered. It is found that the conditions on mesh subdivisions for convergence of some quadrilateral elements guarantees the unisolvence of the shape functions too.

2. Preliminaries. Let there be given a polygonal domain $G \subset \mathbf{R}^{2}$. We decompose $G$ into convex quadrilateral elements with diameters $\leqslant h$. For each quadrilateral $K$ let $h_{K}, h_{K}^{\prime}$ and $\theta_{i}^{K}$ denote the diameter of $K$, the smallest length of the sides of $K$, and the angles associated with the vertices of $K$, respectively. We assume that the decomposition of $G$ satisfies the following regularity conditions (see [1, p. 247]): there exist constants $\sigma^{\prime}$ and $\gamma$ such that

$$
h_{K} \leqslant \sigma^{\prime} h_{K}^{\prime}, \quad \max _{1 \leqslant i \leqslant 4}\left|\cos \theta_{i}^{K}\right| \leqslant \gamma<1
$$

uniformly with respect to $h$ and for all elements $K$.

Let $K$ be a convex quadrilateral with the vertices $p_{i}=\left(x_{i}, y_{i}\right), 1 \leqslant i \leqslant 4$, and the midpoints $p_{i i+1}$ at the sides $\overline{p_{i} p_{i+1}}, 1 \leqslant i \leqslant 4(\bmod 4)$. Let $\hat{K}=[-1,1] \times[-1,1]$ be the reference square with the vertices $\hat{p}_{i}, 1 \leqslant i \leqslant 4$. Then, there exists a unique mapping $F_{K} \in Q_{1}(\hat{K})$ given by

$$
\begin{array}{r}
x=\frac{1}{4}\left[(1+\xi)(1+\eta) x_{1}+(1-\xi)(1+\eta) x_{2}\right. \\
\left.+(1-\xi)(1-\eta) x_{3}+(1+\xi)(1-\eta) x_{4}\right], \\
y=\frac{1}{4}\left[(1+\xi)(1+\eta) y_{1}+(1-\xi)(1+\eta) y_{2}\right. \\
\left.+(1-\xi)(1-\eta) y_{3}+(1+\xi)(1-\eta) y_{4}\right]
\end{array}
$$

such that

$$
F_{K}\left(\hat{p}_{i}\right)=p_{i}, \quad 1 \leqslant i \leqslant 4, \quad F_{K}(\hat{K})=K,
$$

where $Q_{r}(\hat{K})$ denotes the space of all polynomials on $\hat{K}$ with highest degree $r$ in each of the variables $\xi$ and $\eta$.

Denoting the Jacobian of the mapping $F_{K}$ by

$$
J_{K}(\xi, \eta)=\left|\begin{array}{ll}
\frac{\partial x}{\partial \xi} & \frac{\partial x}{\partial \eta} \\
\frac{\partial y}{\partial \xi} & \frac{\partial y}{\partial \eta}
\end{array}\right|,
$$

it is known [1] that under the regularity assumption of the decomposition the following estimates hold:

$$
C_{1} h_{K}^{2} \leqslant J_{K} \leqslant C_{2} h_{K}^{2}
$$

Here and later by $C_{i}, C$ are meant generic constants independent of $h ; C$ may have different values at different places.

To each function $v(x, y)$ defined on $K$ we associate $\hat{v}(\xi, \eta)$ by

$$
\hat{v}(\xi, \eta)=v(x(\xi, \eta), y(\xi, \eta)) \text { or } \hat{v}=v \cdot F_{K} .
$$

The following lemmas are needed which can easily be derived from the inequality (4), the inverse property and the internolation theory on the reference square $\hat{K}$. 

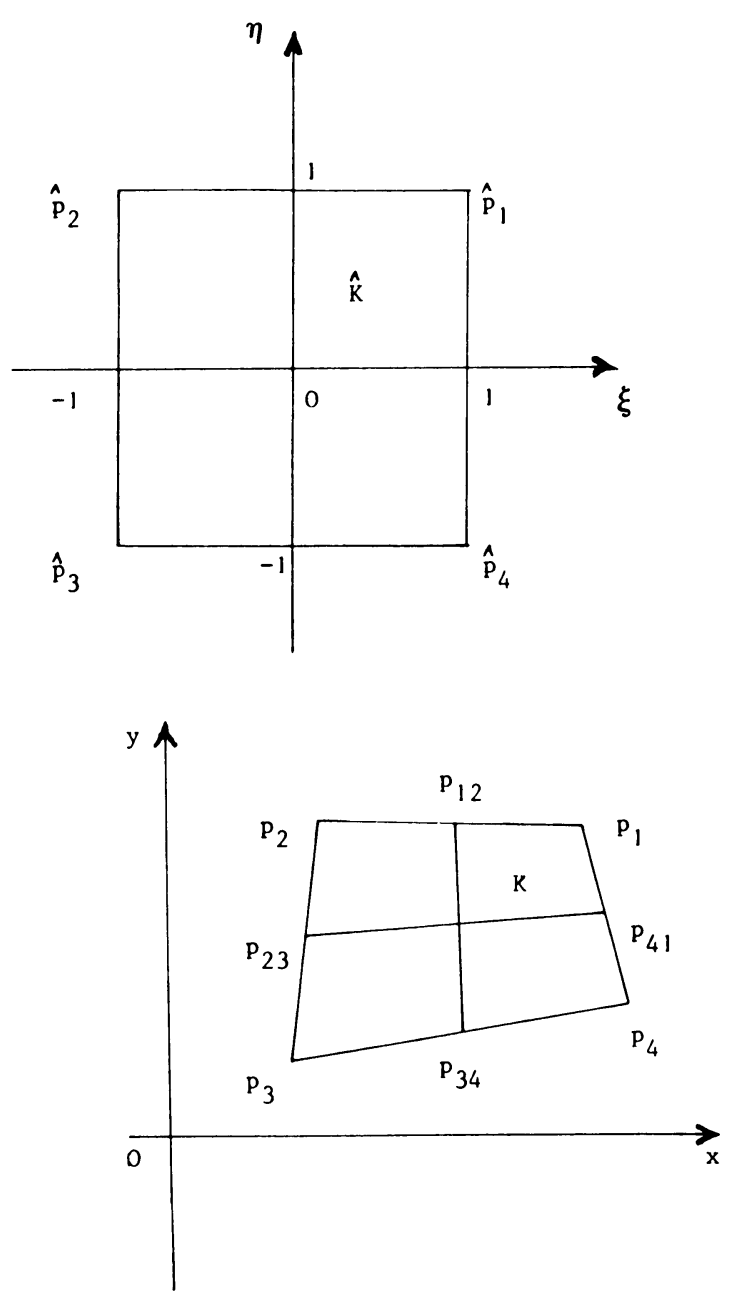

FIGURE 1

LEMMA 1. Let $v \in H^{1}(K)$, and $v$ be associated to $v$ by the relation (5). Then

(6)

$$
|\hat{v}|_{0, \hat{K}} \leqslant C h_{K}^{-1}|v|_{0, K}, \quad|\hat{v}|_{1, \hat{K}} \leqslant C|v|_{1, K} .
$$

LEMMA 2. For any function $v \in Q_{r}(K)$ the inverse inequalities hold:

$$
|v|_{t+1, K} \leqslant C h_{K}^{-1}|v|_{t, K}, \quad t=0,1, \ldots
$$

Next, for every function $\hat{v} \in L^{2}(\hat{K})$ define the operator

$$
\hat{P}_{0}: \hat{v} \rightarrow \frac{1}{4} \int_{\hat{K}} \hat{v} d \xi d \eta,
$$

and let the operator $P_{0}$ be such that

$$
\left(P_{0} v\right)^{\hat{n}}=\hat{P}_{0} \hat{v} \quad \text { for } v=\hat{v} \cdot F_{K}^{-1} .
$$

Therefore,

$$
P_{0} v=\frac{1}{4} \int_{K} J_{K}^{-1} v d x d y
$$


Notice that for the case of a parallelogram $K$, the Jacobian $J_{K}$ is a constant

$$
J_{K}=\frac{|K|}{4}, \quad|K|=\int_{K} 1 d x d y,
$$

so that

$$
P_{0} v=\frac{1}{|K|} \int_{K} v d x d y,
$$

which shows that $P_{0} v$ is the mean value of $v$ over $K$. In general, for quadrilateral $K$, the equality (12) does not hold.

Lemma 3. Let $v \in H^{1}(K)$. Then

$$
\begin{gathered}
\left\|v-P_{0} v\right\|_{0, K} \leqslant C h_{K}|v|_{1, K}, \\
\int_{\partial K}\left(v-P_{0} v\right)^{2} d s \leqslant C h_{K}|v|_{1, K}^{2} .
\end{gathered}
$$

3. Unisolvence of the 8 d.o.f. Quadrilateral Element. We begin with the study of the 8 d.o.f. (degrees of freedom) quadrilateral element used in [3]. The shape function on each element $K$ has the form

$$
u=a_{1}+a_{2} x+a_{3} y+a_{4} x^{2}+a_{5} x y+a_{6} y^{2}+a_{7} x^{2} y+a_{8} x y^{2},
$$

determined by 8 nodal parameters, namely the function values $u_{i}(1 \leqslant i \leqslant 4)$ at the vertices and $u_{i i+1}(1 \leqslant i \leqslant 4, \bmod 4)$ at the midpoints of the sides of $K$.

Let us first discuss the problem of unisolvence of the shape function (15) by the set of nodal parameters $u_{i}$ and $u_{i i+1}(1 \leqslant i \leqslant 4$, mod 4). It is easily verified that for any parallelogram $K$ the shape function (15) is unisolvent since the coefficient matrix $M_{K}$ of the linear equations for determining the unknowns $a_{i}(1 \leqslant i \leqslant 8)$ in (15) is nonsingular, that is,

$$
\operatorname{det}\left(M_{K}\right) \neq 0 .
$$

For general quadrilaterals we give a sufficient condition for the unisolvence of (15) as follows:

Condition (A). The distance $d_{K}$ between the midpoints of the diagonals of $K$ is of order $o\left(h_{k}\right)$.

Evidently, $d_{K}=0$ if and only if $K$ is a parallelogram.

LEMMA 5. Under Condition (A) the shape function (15) is unisolvent for small mesh sizes $h_{K}$.

Proof. Let there be given a quadrilateral $K$. Suppose that one of the vertices of $K$, say $p_{4}$, is located at the origin of the coordinate system. We associate $K$ with a parallelogram $K^{\prime}$ such that three vertices of $K^{\prime}$ coincide with those of $K: p_{i}^{\prime}=p_{i}$ $(i=2,3,4)$ and the vertex $p_{1}^{\prime}$ differs from $p_{1}$ by

$$
x_{1}^{\prime}=x_{1}+\varepsilon_{x} h_{K}, \quad y_{1}^{\prime}=y_{1}+\varepsilon_{y} h_{K} .
$$

Condition (A) implies that

$$
\varepsilon_{x}=o(1), \quad \varepsilon_{y}=o(1) .
$$


Then, we make the transformation of coordinates

$$
g_{K}: \bar{x}=x / h_{K}, \quad \bar{y}=y / h_{K},
$$

so that

$$
g_{K}(K)=\bar{K}, \quad g_{K}\left(K^{\prime}\right)=\bar{K}^{\prime},
$$

with the change of vertices:

$$
p_{i} \rightarrow g_{K}\left(p_{i}\right)=\bar{p}_{i}, \quad p_{i}^{\prime} \rightarrow g_{K}\left(p_{i}^{\prime}\right)=\bar{p}_{i}^{\prime}, \quad 1 \leqslant i \leqslant 4 .
$$

Obviously,

$$
\bar{p}_{i}=\bar{p}_{i}^{\prime} \quad(i=2,3,4), \quad \bar{p}_{1}=\bar{p}_{1}^{\prime}+\left(\varepsilon_{x}, \varepsilon_{y}\right) .
$$

Thus, the quadrilateral $\bar{K}$ may be viewed as a deformation of the parallelogram $\overline{K^{\prime}}$ with only the first vertex $\bar{p}_{1}$ of $\bar{K}$ differing slightly from $\bar{p}_{1}^{\prime}$ of $\bar{K}^{\prime}$.

By virtue of the mapping (18) and the regularity assumption of the decomposition, the coordinates of the vertices $\bar{p}_{i}^{\prime}$ of $\bar{K}^{\prime}$ satisfy the following restrictions:

$$
\begin{gathered}
\frac{1}{\sigma^{\prime 2}} \leqslant \bar{x}_{i}^{\prime 2}+\bar{y}_{i}^{\prime 2} \leqslant 1, \quad i=1,2,3, \\
\bar{x}_{4}^{\prime 2}+\bar{y}_{4}^{\prime 2}=0 .
\end{gathered}
$$

As stated above, for any parallelogram $\overline{K^{\prime}}$ the coefficient matrix $M_{\overline{K^{\prime}}}$ for determining the shape function (15) on $\overline{K^{\prime}}$ is nonsingular:

$$
\operatorname{det}\left(M_{\bar{K}^{\prime}}\right) \neq 0 \text {. }
$$

Since the determinant $\operatorname{det}\left(M_{\bar{K}^{\prime}}\right)$, depending on the coordinates of the vertices and the midpoints of the sides of $\bar{K}^{\prime}$, is a continuous function of the variables $\bar{x}_{i}^{\prime}, \bar{y}_{i}^{\prime}$ $(i=1,2,3)$, and the latter vary in the closed set defined by (20), it follows from (22) that

$$
\left|\operatorname{det}\left(M_{\overline{K^{\prime}}}\right)\right| \geqslant C_{1}>0
$$

independently of $h_{K}$.

On the other hand, for any fixed parallelogram $\bar{K}^{\prime}$, the determinant $\operatorname{det}\left(M_{\bar{K}}\right)$ may be viewed as a polynomial in two variables $\varepsilon_{x}, \varepsilon_{y}$, the perturbations defined by (19), with the constant term $\operatorname{det}\left(M_{\bar{K}^{\prime}}\right)$. Since $\varepsilon_{x}=o(1), \varepsilon_{y}=o(1)$ for $h_{K} \rightarrow 0$ and using the inequality (23), we deduce that

$$
\left|\operatorname{det}\left(M_{\bar{K}}\right)\right| \geqslant C_{2}>0
$$

for small meshes. Noting that

$$
\operatorname{det}\left(M_{K}\right)=h_{K}^{14} \operatorname{det}\left(M_{\bar{K}}\right),
$$

we conclude that the shape function (15) is unisolvent on $\bar{K}$, and hence on $K$ for small meshes. Thus Lemma 5 is proved.

LEMMA 6. Let a convex quadrilateral $K$ be decomposed into $2^{2 n}$ elements by dividing the sides of $K$ in $2^{n}$ equal segments. Then the resulting elements satisfy Condition (A) for large $n$.

The proof is straightforward and therefore omitted.

It is worth mentioning that in many applications a bisection strategy is used for mesh generations, if there is no special information available on how to choose the 
mesh sizes. In such cases, by Lemma 6, Condition (A) is satisfied automatically. In the following it is always assumed that the shape function is unisolvent by its degrees of freedom.

4. Convergence of the 8 d.o.f. Quadrilateral Element. Now we are in a position to prove the convergence of the element (15). Our tool is Stummel's generalized patch test [6], [7], which together with the approximability condition (the latter is satisfied by (15) and any reasonable finite element approximation) provides a necessary and sufficient condition for convergence of nonconforming approximations applied to a large class of general elliptic boundary value problems.

Let $V_{h}$ be the finite element spaces of all functions defined on $\bar{G}$, whose restrictions to each element $K$ are the shape functions of (15). For a second order problem, the generalized patch test consists in showing that, for every bounded sequence $u_{h} \in V_{h}$ and for $h \rightarrow 0$, the following relation

$$
T_{r}\left(\psi, u_{h}\right)=\sum_{K} \int_{\partial K} \psi u_{h} N_{r} d s \rightarrow 0, \quad r=1,2
$$

holds for all test functions $\psi \in C_{0}^{\infty}(G)\left(\psi \in C_{0}^{\infty}\left(\mathbf{R}^{2}\right)\right.$ in case of Dirichlet boundary conditions), where $N_{r}$ denote the components of the unit outward normal vector on the boundary of $K$.

THEOREM 1. The 8 d.o.f. quadrilateral element (15) passes the generalized patch test (25) and hence converges.

Proof. We rewrite the bilinear form $T_{r}\left(\psi, u_{h}\right)$ as follows

$$
T_{r}\left(\psi, u_{h}\right)=\sum_{K} \sum_{F \in \partial K} \int_{F} \psi u_{h} N_{r} d s
$$

and consider first the case where $F \subset \partial K$ is a common side of two adjacent quadrilaterals $K$ and $K^{\prime}$, and second the case that $F \subset \partial K$ is a portion of the boundary $\partial G$.

In the first case, let us denote by $u_{h}^{K}$ and $u_{h}^{K^{\prime}}$ the restrictions of the function $u_{h}$ to $K$ and $K^{\prime}$, respectively. Then, since $u_{h}^{K}-u_{h}^{K^{\prime}}$ is a polynomial of third degree in one variable on $F$ vanishing at the endpoints and the midpoint of $F$, Simpson's rule gives

$$
\int_{F}\left(u_{h}^{K}-u_{h}^{K^{\prime}}\right) d s=0
$$

For every function $v \in L^{2}(F)$, let

$$
P_{0}^{F} v=\frac{1}{|F|} \int_{F} v d s, \quad|F|=\int_{F} d s,
$$

be the mean value of $v$ over $F$. Equality (27) implies that the mean values of $u_{h}$ are continuous on $F$ and, consequently, the two corresponding integrals $\int_{F} P_{0}^{F} \psi u_{h}^{K} N_{r}^{K} d s$ and $\int_{F} P_{0}^{F} \psi u_{h}^{K^{\prime}} N_{r}^{K^{\prime}} d s$ cancel.

In the second case $F \subset \partial G$, the integral

$$
\int_{F} P_{0}^{F} \psi u_{h} N_{r} d s=0
$$


since $P_{0}^{F} u_{h}=0$ for Dirichlet boundary conditions and $\psi \in C_{0}^{\infty}(G)$ otherwise. Therefore,

$$
\sum_{K} \sum_{F \subset \partial K} \int_{F} P_{0}^{F} \psi u_{h} N_{r} d s=0
$$

Similarly, we have

$$
\sum_{K} \sum_{F \subset \partial K} \int_{F}\left(\psi-P_{0}^{F} \psi\right) P_{0}^{F} u_{h} N_{r} d s=0
$$

so that

$$
T_{r}\left(\psi, u_{h}\right)=\sum_{K} \sum_{F \subset \partial K} \int_{F} R_{0}^{F} \psi R_{0}^{F} u_{h} N_{r} d s
$$

where

$$
R_{0}^{F} v=v-P_{0}^{F} v \text {. }
$$

We now estimate the integrals involved in the right-hand side of (31). For every function $v \in L^{2}(K)$ it is easily seen that

$$
\int_{F}\left(R_{0}^{F} v\right)^{2} d s \leqslant \int_{F}\left(v-P_{0}^{K} v\right)^{2} d s
$$

where

$$
P_{0}^{K} v=\frac{1}{4} \int_{K} J_{K}^{-1} v d x d y
$$

as defined in (10). Applying Lemma 3 to (33) and Schwarz's inequality, we obtain

$$
\left|\int_{F} R_{0}^{F} \psi R_{0}^{F} u_{h}^{K} N_{r}^{K} d s\right| \leqslant C h_{K}|\psi|_{1, K}\left|u_{h}\right|_{1, K},
$$

and so

$$
\left|T_{r}\left(\psi, u_{h}\right)\right| \leqslant C h|\psi|_{1}\left|u_{h}\right|_{1, h},
$$

where the seminorm $|\cdot|_{1, h}$ is defined by

$$
|\cdot|_{1, h}^{2}=\sum_{K}|\cdot|_{1, K}^{2}
$$

Hence, the generalized patch test (25) is satisfied.

Remark 1. The element (15) passes the patch test.

Remark 2. The element (15) can also be used for approximations of eigenvalue problems, since the finite element spaces $V_{h}$ satisfy the strong continuity condition (see [7]) on interelement boundaries too.

5. Two 12 d.o.f. Convergent Quadrilateral Elements. Two 12 d.o.f. quadrilateral elements have been studied in [3]. The shape functions of these two elements have, respectively, the form

$$
\begin{aligned}
u= & a_{1}+a_{2} x+a_{3} y+a_{4} x^{2}+a_{5} x y+a_{6} y^{2}+a_{7} x^{2} y+a_{8} x y^{2} \\
& +a_{9} x^{3}+a_{10} y^{3}+a_{11} x^{3} y+a_{12} x y^{3}, \\
u= & a_{1}+a_{2} x+a_{3} y+a_{4} x^{2}+a_{5} x y+a_{6} y^{2}+a_{7} x^{2} y+a_{8} x y^{2} \\
& +\left(x^{2}-a^{2}\right)\left(y^{2}-b^{2}\right)\left(\gamma_{1}+\gamma_{2} x+\gamma_{3} y+\gamma_{4} x y\right),
\end{aligned}
$$


where $2 a$ and $2 b$ in (36) are the lengths of the medians of the quadrilateral $K$. The 12 coefficients involved in (35), (36) are determined by the same set of 8 nodal parameters as for the element (15). The remaining indetermined 4 coefficients are used as internal d.o.f. which are condensed at the element level. Hence the stiffness matrices are of dimensions 8 by 8 . We notice that Condition (A) is still sufficient for the unisolvence of the two shape functions (35) and (36), since the last 4 coefficients in (35) and (36) may be considered as internal d.o.f.

As mentioned in [3], the elmenets (35) and (36) pass the patch test for rectangular meshes but do not pass it for general quadrilaterals. Nevertheless, a trapezoidal membrane problem has been successfully solved using these two quadrilateral elements in conjunction with a bisection scheme for mesh generations. Now we give a mathematical analysis of the two elements to justify the numerical observations of Sander and Beckers in [3].

THEOREM 2. Under Condition (A) the quadrilateral elements (35) and (36) pass the generalized patch test (25) and hence converge.

Proof. Let us first consider the element (35). The bilinear form $T_{r}\left(\psi, u_{h}\right)$ may be written as follows:

$$
\begin{aligned}
T_{r}\left(\psi, u_{h}\right)= & \sum_{K} \sum_{F \subset \partial K} \int_{F} \psi u_{h} N_{r} d s=\sum_{K} \sum_{F \subset \partial K} \int_{F} R_{0}^{F} \psi R_{0}^{F} u_{h} N_{r} d s \\
& +\sum_{K} \sum_{F \subset \partial K} \int_{F} R_{0}^{F} \psi P_{0}^{F} u_{h} N_{r} d s+\sum_{K} \sum_{F \subset \partial K} \int_{F} P_{0}^{F} \psi u_{h} N_{r} d s \\
= & T^{(1)}+T^{(2)}+T^{(3)} .
\end{aligned}
$$

We estimate each of the terms $T^{(i)}(i=1,2,3)$.

(i) Using the same argument as in the proof of Theorem 1, we get immediately

$$
\left|T^{(1)}\right| \leqslant \sum_{K} \sum_{F \subset \partial K}\left|\int_{F} R_{0}^{F} \psi R_{0}^{F} u_{h} N_{r} d s\right| \leqslant C h|\psi|_{1}\left|u_{h}\right|_{1, h} .
$$

(ii) The approximation $u_{h}$ is a polynomial of fourth degree in one variable on every side $F$, e.g. $F=\overline{p_{1} p_{2}}$, Simpson's rule then gives

$$
\begin{aligned}
P_{0}^{F} u_{h}= & \frac{1}{6}\left(u_{h}\left(p_{1}\right)+4 u_{h}\left(p_{12}\right)+u_{h}\left(p_{2}\right)\right) \\
& -\frac{1}{120}\left(x_{1}-x_{2}\right)\left(y_{1}-y_{2}\right)\left[a_{11}\left(x_{1}-x_{2}\right)^{2}+a_{12}\left(y_{1}-y_{2}\right)^{2}\right] \\
= & S_{1}^{F} u_{h}+S_{2}^{F} u_{h} .
\end{aligned}
$$

The first term $S_{1}^{F} u_{h}$ is continuous on $F$, so that we have

$$
\sum_{K} \sum_{F \subset \partial K} \int_{F} R_{0}^{F} \psi S_{1}^{F} u_{h} N_{r} d s=0
$$

which implies

$$
T^{(2)}=\sum_{K} \sum_{F \subset \partial K} \int_{F} R_{0}^{F} \psi S_{2}^{F} u_{h} N_{r} d s
$$


Since

$$
a_{11}=\frac{1}{6} \frac{\partial^{4} u_{h}}{\partial x^{3} \partial y}, \quad a_{12}=\frac{1}{6} \frac{\partial^{4} u_{h}}{\partial x \partial y^{3}}
$$

it follows that

$$
\left|a_{11}\right| \leqslant \frac{1}{6|K|^{1 / 2}}\left|u_{h}\right|_{4, K}, \quad\left|a_{12}\right| \leqslant \frac{1}{6|K|^{1 / 2}}\left|u_{h}\right|_{4, K} .
$$

The inverse inequalities in Lemma 2 then give

$$
\left|a_{11}\right| \leqslant C h_{K}^{-4}\left|u_{h}\right|_{1, K}, \quad\left|a_{12}\right| \leqslant C h_{K}^{-4}\left|u_{h}\right|_{1, K} .
$$

By virtue of the form of $S_{2}^{F} u_{h}$ in (39), application of (41) yields

$$
\left|S_{2}^{F} u_{h}\right| \leqslant C\left|u_{h}\right|_{1, K}
$$

so that

$$
\left|T^{(2)}\right| \leqslant \sum_{K} \sum_{F \subset \partial K}\left|\int_{F} R_{0}^{F} \psi S_{2}^{F} u_{h} N_{r} d s\right| \leqslant C h|\psi|_{1}\left|u_{h}\right|_{1, h} .
$$

(iii) We now analyze the term $T^{(3)}$. By (39),

$$
T^{(3)}=\sum_{K} \sum_{F \subset \partial K} \int_{F} P_{0}^{F} \psi u_{h} N_{r} d s=\sum_{K} \sum_{F \subset \partial K} P_{0}^{F} \psi S_{2}^{F} u_{h}|F| N_{r}^{F}
$$

since

$$
\sum_{K} \sum_{F \subset \partial K} P_{0}^{F} \psi S_{1}^{F} u_{h}|F| N_{r}^{F}=0
$$

Setting $F_{i}=\overline{p_{i} p_{i+1}}(1 \leqslant i \leqslant 4 \bmod 4)$ and $r=1$, we have

$$
\left|F_{i}\right| N_{1}^{F_{i}}=y_{i+1}-y_{i} \text {. }
$$

Thus, for the two opposite sides $F_{1}$ and $F_{3}$ of $K$, the sum of the corresponding terms in (43) has the form

$$
\begin{aligned}
& P_{0}^{F_{1}} \psi S_{2}^{F_{1}} u_{h}\left|F_{1}\right| N_{1}^{F_{1}}+P_{0}^{F_{3}} \psi S_{2}^{F_{3}} u_{h}\left|F_{3}\right| N_{1}^{F_{3}} \\
& =\frac{1}{120}\left\{\left[P_{0}^{F_{1}} \psi\left(x_{1}-x_{2}\right)^{3}\left(y_{1}-y_{2}\right)^{2}-P_{0}^{F_{3}} \psi\left(x_{4}-x_{3}\right)^{3}\left(y_{4}-y_{3}\right)^{2}\right] a_{11}\right. \\
& \left.\quad+\left[P_{0}^{F_{1}} \psi\left(x_{1}-x_{2}\right)\left(y_{1}-y_{2}\right)^{4}-P_{0}^{F_{3}} \psi\left(x_{4}-x_{3}\right)\left(y_{4}-y_{3}\right)^{4}\right] a_{12}\right\}
\end{aligned}
$$

Noting that

$$
\begin{aligned}
\left|P_{0}^{F_{1}} \psi-P_{0}^{F_{3}} \psi\right|= & \left|\int_{0}^{1} \psi\left(p_{1}+s\left(p_{2}-p_{1}\right)\right) d s-\int_{0}^{1} \psi\left(p_{3}+s\left(p_{4}-p_{3}\right)\right) d s\right| \\
\leqslant & C|\psi|_{1, K}, \\
& \left|P_{0}^{F_{i}} \psi\right| \leqslant|\psi|_{\infty}, \quad i=1,2,3,4
\end{aligned}
$$

and that Condition (A) yields

$$
x_{1}-x_{2}=x_{4}-x_{3}+o\left(h_{K}\right), \quad y_{1}-y_{2}=y_{4}-y_{3}+o\left(h_{K}\right) \text {, }
$$


we deduce from (41), (45)-(48) that

$$
\begin{aligned}
& \left|P_{0}^{F_{1}} \psi S_{2}^{F_{1}} u_{h}\right| F_{1}\left|N_{1}^{F_{1}}+P_{0}^{F_{3}} \psi S_{2}^{F_{3}} u_{h}\right| F_{3}\left|N_{1}^{F_{3}}\right| \\
& \quad \leqslant C h_{K}|\psi|_{1, K}\left|u_{h}\right|_{1, K}+o\left(h_{K}\right)|\psi|_{\infty}\left|u_{h}\right|_{1, K} .
\end{aligned}
$$

Similarly, for the two opposite sides $F_{2}$ and $F_{4}$ of $K$ we get the corresponding estimate:

$$
\begin{aligned}
& \left|P_{0}^{F_{2}} \psi S_{2}^{F_{2}} u_{h}\right| F_{2}\left|N_{1}^{F_{2}}+P_{0}^{F_{4}} \psi S_{2}^{F_{4}} u_{h}\right| F_{4}\left|N_{1}^{F_{4}}\right| \\
& \quad \leqslant C h_{K}|\psi|_{1, K}\left|u_{h}\right|_{1, K}+o\left(h_{K}\right)|\psi|_{\infty}\left|u_{h}\right|_{1, K}
\end{aligned}
$$

Therefore, for $r=1$ we have

$$
\left|T^{(3)}\right| \leqslant C h|\psi|_{1}\left|u_{h}\right|_{1, h}+o(1)|\psi|_{\infty}\left|u_{h}\right|_{1, h} .
$$

Evidently, the above estimate also holds for $r=2$. Combining the inequalities (38), (42) and (51), we have proved that

$$
\left|T_{r}\left(\psi, u_{h}\right)\right| \leqslant C h|\psi|_{1}\left|u_{h}\right|_{1, h}+o(1)|\psi|_{\infty}\left|u_{h}\right|_{1, h}, \quad r=1,2,
$$

which means that the element (35) passes the generalized patch test (25).

In the same manner it can be proved that also the element (36) passes the test (25).

For the elements (35), (36), the approximability and strong continuity conditions are satisfied. Therefore, according to Stummel's theory [7], we have proved that under Condition (A) the elements (35) and (36), which do not pass the patch test, converge indeed. Consequently, these nonconforming finite elements may be used for approximations of general second order elliptic equations and associated eigenvalue problems.

Remark 1. Condition (A) guarantees not only the unisolvence of the shape functions (35), (36), but also the satisfaction of the generalized patch test. As the mesh is refined, the shape of the quadrilaterals more and more resembles parallelograms; however, the patch test is still violated.

Remark 2. If instead of the function values at mid-side nodes the averages over the corresponding sides of $K$ are used as degrees of freedom, the resulting 12 d.o.f. quadrilateral elements pass the patch test. The convergence can be proved using the same argument as in Section 4.

6. A Divergent 16 d.o.f. Quadrilateral Element. It remains to consider a 16 d.o.f. quadrilateral element whose shape function has the form

$$
\begin{aligned}
u= & a_{1}+a_{2} x+a_{3} y+a_{4} x^{2}+a_{5} x y+a_{6} y^{2}+a_{7} x^{3}+a_{8} x^{2} y+a_{9} x y^{2} \\
& +a_{10} y^{3}+a_{11} x^{4}+a_{12} x^{3} y+a_{13} x y^{3}+a_{14} y^{4}+a_{15} x^{4} y+a_{16} x y^{4}
\end{aligned}
$$

with the 8 nodal parameters as before and 8 internal d.o.f. The element does not pass the patch test even for rectangular meshes. Numerical results in [3] showed that the element does not yield convergent approximations. This is confirmed by the following theorem.

THEOREM 3. The element (53) does not pass the generalized patch test (25) and hence diverges.

Proof. We show that there exists a sequence of trial functions $u_{h} \in V_{h}$ and a test function $\psi$ such that the test (25) does not hold. 
Let a unit square $G=(0,1) \times(0,1)$ be given, and consider a decomposition of $G$ by square meshes with the sizes

$$
h_{n}=\frac{1}{2^{n}}, \quad n=2,3, \ldots
$$

Then choose the fixed subdomain $G_{1}=\left[\frac{1}{4}, \frac{3}{4}\right] \times\left[\frac{1}{4}, \frac{3}{4}\right]$ in $G$. There are $N_{1}=2^{2(n-1)}$ $(n \geqslant 2)$ square elements $K$ in $G_{1}$. Let $K \subset G_{1}$ be described in local coordinates as the set $\left[0, h_{n}\right] \times\left[0, h_{n}\right]$. With respect to these coordinates we define a special sequence of trial functions $u_{h}$ as

$$
u_{h}(x, y)=x\left(h_{n}-x\right)\left(x-\frac{h_{n}}{2}\right)^{2} y, \quad(x, y) \in\left[0, h_{n}\right] \times\left[0, h_{n}\right],
$$

outside $G_{1}$, let $u_{h}=0$.

We further select a test function $\psi \in C_{0}^{\infty}(G)$ such that

$$
\psi \equiv 1 \text { on } G_{1} .
$$

In case of Dirichlet boundary conditions, in addition, choose

$$
\psi \equiv 0 \quad \text { on } \mathbf{R}^{2} \backslash G
$$

Because of the special choices of $u_{h}$ and $\psi$, the bilinear form becomes

$$
T_{r}\left(\psi, u_{h}\right)=\sum_{K \in G} \int_{\partial K} \psi u_{h} N_{r} d s=\frac{1}{120} h_{n}^{6} \cdot \sum_{K \in G_{1}} 1=\frac{1}{480} h_{n}^{4},
$$

and

$$
\left\|u_{h}\right\|_{0, K}^{2}=\frac{1}{30240} h_{n}^{12} .
$$

Using the inverse property, we have

$$
\left|u_{h}\right|_{1, K} \leqslant C h_{n}^{-1}\left\|u_{h}\right\|_{0, K} \leqslant C h_{n}^{5}
$$

and so

$$
\left\|u_{h}\right\|_{1, h}^{2}=\sum_{K}\left\|u_{h}\right\|_{1, K}^{2} \leqslant C h_{n}^{8}\left(1+O\left(h_{n}^{2}\right)\right) .
$$

Consequently,

$$
\frac{T_{r}\left(\psi, u_{h}\right)}{\left\|u_{h}\right\|_{1, h}} \geqslant C\left(1+O\left(h_{n}^{2}\right)\right), \quad C \neq 0,
$$

for $h_{n} \rightarrow 0$. This shows that the element (53) fails to pass the generalized patch test and thus does not converge.

Remark 1. Like Remark 2 in Theorem 2, if the averages over the sides of the quadrilaterals are chosen as d.o.f. instead of the function values at mid-side nodes, the resulting 16 d.o.f. quadrilateral element becomes convergent.

Acknowledgement. The author is very grateful to Professor F. Stummel for suggesting this investigation and for continuous encouragement in the preparation of the paper.

Department of Mathematics

China University of Science and Technology

Hofei, People's Republic of China 
1. P. G. Ciarlet, The Finite Element Method for Elliptic Problems, North-Holland, Amsterdam, 1978.

2. B. M. Irons \& A. Razzaque, Experience With the Patch Test for Convergence of Finite Elements, Proc. Sympos. on Mathematical Foundations of the Finite Element Method with Application to Partial Differential Operators (Baltimore 1972), (A. K. Aziz, ed.), Academic Press, New York, 1972, pp. 557-587.

3. G. SANDER \& P. BeCKers, "The influence of the choice of connectors in the finite element method," Internat. J. Numer. Methods Engrg., v. 11, 1977, pp. 1491-1505.

4. ZHONG-CI SHI, "An explicit analysis of Stummel's patch test examples," Internat. J. Numer. Methods Engrg. (To appear.)

5. F. Stummel, "The limitations of the patch test," Internat. J. Numer. Methods Engrg, v. 15, 1980, pp. $177-188$.

6. F. Stummel, “The generalized patch test,” SIAM J. Numer. Anal., v. 16, 1979, pp. 449-471.

7. F. STUMmel, "Basic compactness properties of nonconforming and hybrid finite element spaces," RAIRO Anal. Numer., v. 4, 1980, pp. 81-115. 